\title{
Feedback min-max model predictive control using a single linear program: Robust stability and the explicit solution
}

\author{
Eric C. Kerrigan ${ }^{\dagger}$ and Jan M. Maciejowski \\ Department of Engineering, University of Cambridge \\ Trumpington Street, Cambridge CB2 1PZ, United Kingdom \\ Tel: +44-(0)1223-332600, Fax: +44-(0)1223-332662 \\ \{eck21,jmm\}@eng.cam.ac.uk, http://www-control.eng.cam.ac.uk
}

\begin{abstract}
SUMMARY
In this paper we introduce a new stage cost and show that the use of this cost allows one to formulate a robustly stable feedback min-max model predictive control problem that can be solved using a single linear program. Furthermore, this is a multi-parametric linear program, which implies that the optimal control law is piecewise affine, and can be explicitly pre-computed so that the linear program does not have to be solved on-line. We assume that the plant model is known, is discrete-time and linear time-invariant, is subject to unknown but bounded state disturbances and that the states of the system are measured. Two numerical examples are presented; one of these is taken from the literature, so that a direct comparison of solutions and computational complexity with earlier proposals is possible. This is a preprint of an article published in International Journal of Robust and Nonlinear Control Copyright (c) 2004 John Wiley \& Sons, Ltd.
\end{abstract}

KEY WORDS: min-max problems, robust control, optimal control, receding horizon control

\section{INTRODUCTION}

The problem of steering a constrained system subject to persistent disturbances to a target set, while also minimising some worst case cost, was considered as early as the 1960s and [12, 13, 16, 19,39] contain some of the first, and perhaps also some of the most insightful, results. In $[12,19]$ set-based solutions to the robust time-optimal problem were presented, but the unsolved problem was how to keep the state evolution inside the target set once it had been reached. The latter problem was solved in $[9,34]$ by requiring that the target set be robustly invariant; once inside the target set the control input is determined by a pre-computed control law that ensures that the state trajectory never leaves the target set. Furthermore, [34] continues by decomposing the state space into simplices and computing an explicit affine expression for the control law in each simplex. All that is required on-line is to determine in which simplex the current state lies and the control input is then given by the pre-computed affine

Contract/grant sponsor: Royal Academy of Engineering, UK.

${ }^{\dagger}$ Royal Academy of Engineering Post-doctoral Research Fellow.

This is a preprint of an article published in International Journal of Robust and Nonlinear Control Copyright (C) 2004 John Wiley \& Sons, Ltd. 
control law. More recent attempts at the control of constrained systems are based on set invariance theory and the reader is referred to $[11,20]$ for an introduction into this field.

In general, solving a min-max problem subject to constraints and disturbances is computationally too demanding for practical implementation. However, various attempts have been made at presenting approximate solutions to this problem and most of these solutions appear to have come from the field of robust model predictive control (MPC) [30, 33]. Usually, MPC schemes obtain on-line the solution to a finite-horizon approximation of the infinite-horizon problem. For a given state only the initial segment of the optimal sequence is implemented; at the next time instant a new measurement is taken and a new finite-horizon min-max problem is solved.

Due to the various assumptions and approximations made, it is difficult to compare different robust MPC schemes with one another. However, most robust MPC schemes can be classified into two categories [33]: (i) open-loop min-max MPC [1, 2, 14, 40], where a single control input sequence (or sequence of perturbations to a given stabilising control law $[25,29]$ ) is used to minimise the worst case cost, and (ii) feedback min-max MPC [4, 5, 21, 24, 28, 36], where the worst case cost is minimised over a sequence of feedback control laws. In general, the open-loop formulation is too conservative and often severely under-estimates the set of feasible trajectories. The feedback MPC formulation was proposed in [31] as an improvement over open-loop MPC.

It is by now well-established that with polytopic disturbance bounds, a linear model and a convex cost, in order to solve feedback min-max MPC problems it is sufficient to consider only the disturbance realisations that take on values at the vertices of the disturbance polytope [36]. However, the number of extreme disturbance realisations typically grows exponentially with the length of the prediction horizon used in MPC.

As an alternative, in $[4,5,21]$ it is proposed that a dynamic- and parametric programming approach be used to obtain an explicit expression for the control law. Provided the stage cost is piecewise affine (e.g. if a 1-norm or $\infty$-norm is used), a piecewise affine expression for the control law can be computed off-line. However, stability is not proven for the stage and terminal costs proposed in $[4,5]$ nor do the costs satisfy the stability conditions given in [32, §3.3] and [33, §4.4].

In Section 2 we define the feedback min-max problem that will be considered and present the main contribution of this paper, which is the introduction of a new type of stage cost (see (10)) such that robust asymptotic stability of a given target set is guaranteed. In Section 3 we review known requirements for a receding horizon controller to be robustly stable, and show how the newlyintroduced stage cost satisfies these requirements. We also point out some advantages of this cost, over the cost proposed in [21]. In Section 4 we show how the finite-horizon feedback min-max problem can be solved as a single linear program (LP), using the results presented in [36], and point out its multi-parametric nature. Two numerical examples are presented in Section 5 and the conclusions are given in Section 6.

\section{PROBLEM FORMULATION}

We consider a discrete-time, linear, time-invariant plant

$$
x_{k+1}=A x_{k}+B u_{k}+w_{k},
$$

where $x_{k} \in \mathbb{R}^{n}$ is the system state, $u_{k} \in \mathbb{R}^{m}$ is the control input and $w_{k} \in \mathbf{W}$ is a persistent disturbance that only takes on values in a polytope $\mathbf{W} \subset \mathbb{R}^{n}$ (a polytope is defined to be a bounded, closed and convex polyhedron - in other words, the sets $\mathbf{W}, \mathbf{X}, \mathbf{U}$ and $\mathbf{T}$, to be defined below, are compact and

This is a preprint of an article published in International Journal of Robust and Nonlinear Control Copyright (C) 2004 John Wiley \& Sons, Ltd. Int. J. Robust Nonlinear Control 2004; 14:395-413

Prepared using rncauth.cls 
convex sets that can be described by a finite number of affine inequalities). It is assumed that the disturbance $w_{k}$ can jump between arbitrary values within $\mathbf{W}$ and that no stochastic description for it is postulated. Therefore, a worst-case approach is taken in this paper. It is assumed that $(A, B)$ is stabilisable and that polytopic constraints on the state and input, that are either due to physical, safety or performance considerations, are also given:

$$
x_{k} \in \mathbf{X}, \quad u_{k} \in \mathbf{U}, \quad \forall k \in \mathbb{N} .
$$

We assume that $\mathbf{W}$ contains the origin and that $\mathbf{X} \subset \mathbb{R}^{n}$ and $\mathbf{U} \subset \mathbb{R}^{m}$ contain the origin in their interiors.

Since a persistent, unknown disturbance is present, it is impossible to drive the state to the origin. Instead, it is only possible to drive the system to a bounded target set contained inside $\mathbf{X}$. The goal is to obtain a (time-invariant) nonlinear feedback control law $u=\kappa(x)$ such that the system is robustly steered to the target set, while also satisfying the state and input constraints, and minimising some worst case cost.

In order to determine a suitable control law an optimal control problem $P_{N}$ (defined below) with horizon $N$ is solved. Let $\mathbf{w}:=\left\{w_{0}, w_{1}, \ldots, w_{N-1}\right\}$ denote a disturbance sequence over the interval 0 to $N-1$. Effective control in the presence of the disturbance requires state feedback [33, §4.6], so that the decision variable in the optimal control problem (for a given initial state) is a control policy $\pi$ defined by

$$
\pi:=\left\{u(0), \mu_{1}(\cdot), \ldots, \mu_{N-1}(\cdot)\right\},
$$

where $u(0) \in \mathbf{U}$ and $\mu_{k}: \mathbf{X} \rightarrow \mathbf{U}, k \in\{1, \ldots, N-1\} ; u(0)$ is a control action (since the current state is known) and each $\mu_{k}(\cdot)$ is a state feedback control law. Let $\phi(k ; x, \pi, \mathbf{w})$ denote the solution to (1) at time $k$ when the state is $x$ at time 0 , the control is determined by policy $\pi\left(u=\mu_{k}(x)\right.$ at event $(x, k)$, i.e. state $x$, time $k$ ) and the disturbance sequence is $\mathbf{w}$.

Given a target set (often also called terminal constraint) $\mathbf{T} \subset \mathbf{X}$, for each initial state $x \in \mathbb{R}^{n}$, let $\Pi_{N}(x)$ denote the set of admissible policies, i.e.

$$
\begin{array}{r}
\Pi_{N}(x):=\left\{\pi \mid u(0) \in \mathbf{U}, \mu_{k}(\phi(k ; x, \pi, \mathbf{w})) \in \mathbf{U}, \phi(k ; x, \pi, \mathbf{w}) \in \mathbf{X}, \phi(N ; x, \pi, \mathbf{w}) \in \mathbf{T},\right. \\
\left.\forall k \in\{1, \ldots, N-1\}, \forall \mathbf{w} \in \mathbf{W}^{N}\right\}
\end{array}
$$

and let

$$
X_{N}:=\left\{x \in \mathbb{R}^{n} \mid \Pi_{N}(x) \neq \emptyset\right\}
$$

denote the set of states that can be robustly steered (steered for all $\mathbf{w} \in \mathbf{W}^{N}:=\overbrace{\mathbf{W} \times \cdots \times \mathbf{W}}^{N \text { times }}$ to the target set $\mathbf{T}$ in $N$ steps, while satisfying all input and state constraints.

In order to define an optimal control problem, a cost $V_{N}(\cdot)$ that is dependent on the policy $\pi$ and current state $x$, but not dependent on $\mathbf{w}$, is defined; the conventional choice is

$$
V_{N}(x, \pi):=\max _{\mathbf{w} \in \mathbf{W}^{N}}\left[\sum_{k=0}^{N-1} L\left(x_{k}, u_{k}\right)+F\left(x_{N}\right)\right]
$$

where $x_{k}:=\phi(k ; x, \pi, \mathbf{w})$ if $k \in\{0, \ldots, N\}, u_{k}:=\mu_{k}(\phi(i ; x, \pi, \mathbf{w}))$ if $k \in\{1, \ldots, N-1\}$ and $u_{0}:=u(0)$.

This is a preprint of an article published in International Journal of Robust and Nonlinear Control Copyright (C) 2004 John Wiley \& Sons, Ltd. 
The target set $\mathbf{T}$, stage cost $L(\cdot)$ and terminal cost $F(\cdot)$ have to satisfy certain conditions in order to ensure that the solution of the feedback min-max optimal control problem, when implemented in a receding horizon fashion, is robustly stabilising. These conditions will be set out in Section 3 .

The feedback min-max optimal control problem $P_{N}$ can now be defined as

$$
P_{N}(x): \quad V_{N}^{*}(x):=\min _{\pi}\left\{V_{N}(x, \pi) \mid \pi \in \Pi_{N}(x)\right\} .
$$

Let $\pi_{N}^{*}(x)$ denote the solution to $P_{N}(x)$, i.e.

$$
\pi_{N}^{*}(x):=\left\{u_{0}^{*}(x), \mu_{1}^{*}(\cdot ; x), \ldots, \mu_{N-1}^{*}(\cdot ; x)\right\}:=\arg \min _{\pi}\left\{V_{N}(x, \pi) \mid \pi \in \Pi_{N}(x)\right\},
$$

where the notation $\mu_{i}^{*}(\cdot ; x)$ shows the dependence of the optimal policy on the current state $x$. It is assumed that $X_{N}$ is non-empty and that a minimiser $\pi_{N}^{*}(x)$ to problem $P_{N}(x)$ exists for all $x \in X_{N}$ (it will become clear in Section 4 that this assumption is justified).

It should be noted that the solution to problem $P_{N}$ is frequently not unique - that is, there can be a whole set of minimisers, from which one must be selected. Thus the time-invariant, set-valued receding horizon control (RHC) law $\kappa_{N}: X_{N} \rightarrow 2^{U}\left(2^{U}\right.$ is the set of all subsets of $U$ ) is defined by the first element of $\pi_{N}^{*}(x)$ :

$$
\kappa_{N}(x):=u_{0}^{*}(x), \forall x \in X_{N} .
$$

Typically, but not always, $u_{0}^{*}(x)$ is a singleton.

The feedback min-max problem $P_{N}$ defined in (6) is an infinite dimensional optimisation problem and impossible to solve directly. However, methods for solving $P_{N}$ using finite dimensional optimisation techniques have been proposed in $[4,5,21,36]$ and this paper can be seen as an immediate extension of [36].

Before proceeding, some comments regarding the choice of stage cost are in order. Robust stability can be guaranteed if the stage cost

$$
L(x, u):=\left\{\begin{array}{ll}
\|Q x\|_{p}+\|R u\|_{p} & \text { if }(x, u) \in(\mathbf{X} \backslash \mathbf{T}) \times \mathbf{U} \\
0 & \text { if }(x, u) \in \mathbf{T} \times \mathbf{U}
\end{array},\right.
$$

proposed in [21, 32], is used. Though this choice of cost solves the stability problem, it should be noted that (9) is not continuous (on the boundary of $\mathbf{T}$ ). The use of such a discontinuous stage cost is a major obstacle to implementation using standard solvers for linear, quadratic, semi-definite or other smooth, convex nonlinear programming problems. A new cost (defined below) is proposed as an alternative that solves the problem of obtaining a continuous stage cost that can be implemented using smooth, convex programming solvers, while still guaranteeing robust stability of the closed-loop system.

We introduce here a new type of stage cost:

$$
L(x, u):=\min _{y \in \mathbf{T}}\|Q(x-y)\|_{p}+\|R(u-K x)\|_{p},
$$

where $Q \in \mathbb{R}^{n \times n}$ and $R \in \mathbb{R}^{m \times m}$ are weights, $K \in \mathbb{R}^{m \times n}$ is a linear feedback gain and $\mathbf{T} \subset \mathbb{R}^{n}$ is the target set and is a polytope containing the origin in its interior (recall that a polytope was defined to be a compact polyhedron, hence the minimum in (10) exists); the choice and role of the feedback gain $K$ and target set $\mathbf{T}$ will be discussed in more detail in Section 3. We will show in Section 4 that if $p=1$ or $p=\infty$, then the use of this stage cost allows the robustly stable feedback min-max MPC problem to be solved using a single LP. Furthermore, we will show that this LP is in fact a multi-parametric LP (mp-LP), that allows the RHC law $\kappa_{N}(\cdot)$ to be pre-computed off-line along the lines developed in $[3,6,8]$, and from which it follows that this law is in fact piecewise affine. These facts make robust MPC/RHC, using the stage cost (10), a viable proposition for some realistic problems.

This is a preprint of an article published in International Journal of Robust and Nonlinear Control Copyright (C) 2004 John Wiley \& Sons, Ltd. Int. J. Robust Nonlinear Control 2004; 14:395-413

Prepared using rncauth.cls 
Remark 1. A similar stage cost to (10) was independently proposed in [27] and briefly discussed within the context of guaranteeing robust stability of a new type of MPC scheme. The stage cost proposed in [27] is $L(x, u):=(1 / 2)\left\|x-\operatorname{Proj}_{\mathbf{T}}(x)\right\|_{2}^{2}+(1 / 2)\|u-K x\|_{2}^{2}$, where $\operatorname{Proj}_{\mathbf{T}}(x)$ denotes the orthogonal projection of $x$ onto $\mathbf{T}$. The difference between this stage cost and (10) is minor, but the formulation in (10) is perhaps more natural. More importantly, the MPC scheme proposed in [27] is fundamentally different from the feedback min-max MPC scheme considered here and [27] only briefly discusses the properties of their proposed stage cost. This paper makes a contribution by analysing and discussing the properties of (10) in detail with regards to its use in feedback min-max MPC.

Remark 2. This paper investigates the use of (10) in solving $P_{N}$ using the method proposed in [36]. Though not discussed here, it is possible to use (10) in order to guarantee stability if $P_{N}$ is solved using the methods described in [4, 5, 21].

\section{REQUIREMENTS FOR ROBUST STABILITY}

It is well-known that, for an MPC/RHC law that assumes a finite horizon, an arbitrary choice of terminal constraint, stage cost and terminal cost does not guarantee stability of the closed-loop system. In the absence of state disturbances, conventional MPC/RHC schemes employ a terminal cost $F(x):=\|P x\|$, that is a control Lyapunov function inside $\mathbf{T}$, in order to guarantee robust stability of the origin for the closed-loop system [32, 33]. However, if the interior of $\mathbf{W}$ is non-empty and the disturbance is persistent, then one can easily show that there does not exist a so-called robust control Lyapunov function $[32,33]$ in a neighbourhood of the origin. Since it is no longer possible to drive the system to the origin, but only to some set containing the origin, the conventional choice of stage and terminal cost cannot guarantee stability or convergence [32, §3.3.2] and a new type of stage and terminal cost is needed.

\subsection{Sufficient conditions for stability}

Before proceeding to set up conditions for robust stability some definitions, taken from [21], are in order. If $d(z, Z):=\inf _{y \in Z}\|z-y\|$ for any set $Z \subset \mathbb{R}^{n}$ and $\|\cdot\|$ denotes any norm, then the set $\mathbf{T}$ is robustly stable iff, for all $\varepsilon>0$, there exists a $\delta>0$ such that $d\left(x_{0}, \mathbf{T}\right) \leq \delta$ implies $d\left(x_{i}, \mathbf{T}\right) \leq \varepsilon$, for all $i \geq 0$ and all admissible disturbance sequences. The set $\mathbf{T}$ is robustly asymptotically (finite-time) attractive with domain of attraction $X$ iff for all $x_{0} \in X, d\left(x_{i}, \mathbf{T}\right) \rightarrow 0$ as $i \rightarrow \infty$ (there exists a time $M$ such that $x_{i} \in \mathbf{T}$ for all $i \geq M$ ) for all admissible disturbance sequences. The set $\mathbf{T}$ is robustly asymptotically (finite-time) stable with domain of attraction $X$ iff it is robustly stable and robustly asymptotically (finite-time) attractive with domain of attraction $X$.

Consider now the following assumptions, adapted from [21, 36, 37]:

A1: The terminal constraint set $\mathbf{T} \subset \mathbf{X}$ is a polytope containing the origin in its interior. A linear, time-invariant control law $K: \mathbb{R}^{n} \rightarrow \mathbb{R}^{m}$ is given such that the terminal constraint set $\mathbf{T}$ is disturbance invariant [23] for the closed-loop system, i.e. $(A+B K) x+w \in \mathbf{T}$ for all $x \in \mathbf{T}$ and all $w \in \mathbf{W}$. In addition, $K x \in \mathbf{U}$ for all $x \in \mathbf{T}$.

A2: The terminal cost $F(x):=0$ for all $x \in \mathbb{R}^{n}$.

A3: The stage cost $L(x, u):=0$ if $x \in \mathbf{T}$ and $u=K x$.

A4a: $L(\cdot)$ is continuous over $\mathbf{X} \times \mathbf{U}$ and there exists a $c>0$ such that $L(x, u) \geq c(d(x, \mathbf{T}))$ for all $(x, u) \in(\mathbf{X} \backslash \mathbf{T}) \times \mathbf{U}$.

A4b: $L(\cdot)$ is continuous over $(\mathbf{X} \backslash \mathbf{T}) \times \mathbf{U}$ and there exists a $c>0$ such that $L(x, u) \geq c\|x\|$ for all

This is a preprint of an article published in International Journal of Robust and Nonlinear Control Copyright (C) 2004 John Wiley \& Sons, Ltd.

Prepared using rncauth.cls 
$(x, u) \in(\mathbf{X} \backslash \mathbf{T}) \times \mathbf{U}$.

$\mathrm{A} 1, \mathrm{~A} 2, \mathrm{~A} 3, \mathrm{~A} 4 \mathrm{a}$ and A4b satisfy the assumptions on the stage cost, terminal cost and terminal constraint given in $[32, \S 3.3]$ and $[33, \S 4.4]$. Hence, one can follow a standard procedure of using the optimal value function as a candidate Lyapunov function $[32,33]$ and show that:

Theorem 1. If $A 1, A 2, A 3$ and $A 4 a$ (and $A 4 b$ ) hold, then $\mathbf{T}$ is robustly asymptotically (finite-time) stable for the closed-loop system $x_{k+1}=A x_{k}+B \kappa_{N}\left(x_{k}\right)+w_{k}$ with a region of attraction $X_{N}$.

Remark 3. Consider also the "dual-mode" control law

$$
\Gamma(x):= \begin{cases}\kappa_{N}(x) & \text { if } x \in X_{N} \backslash \mathbf{T} \\ K x & \text { if } x \in \mathbf{T}\end{cases}
$$

where $\kappa_{N}(\cdot)$ is defined in (8). If $\mathbf{T}, K, F(\cdot)$ and $L(\cdot)$ are chosen such that assumptions A1, A2, A3 and $A 4$ are satisfied, then $\Gamma(\cdot)$ is clearly also a robustly stabilising control law, by Theorem 1.

Remark 4. If $A 1$ and $A 2$ are satisfied and (10) is used for the stage cost with $R$ being non-singular, then $\kappa_{N}(x)=K x$ for all $x \in \mathbf{T}$. This is not guaranteed if $R$ is singular.

In [33, \$4.6.3] and [36] it is argued that one need only consider the set of extreme disturbance realisations if the following assumption holds in addition to those given above:

A5: $L(\cdot)$ is convex over $\mathbf{X} \times \mathbf{U}$.

It is shown in [36] how, provided A1, A2, A3, A4a (and A4b) and A5 hold, one can associate a different control input sequence with each extreme disturbance realisation and, using a causality constraint that prevents the optimiser from assuming knowledge of future disturbances, one can compute a control input $u \in \kappa_{N}(x)$ on-line using standard finite-dimensional convex programming solvers. However, in [33, §4.6.3] and [36], an exact expression for the stage cost that allows one to implement the proposed method is not given; only general conditions on $L(\cdot)$ as in $\mathrm{A} 3, \mathrm{~A} 4 \mathrm{a}$ and $\mathrm{A} 4 \mathrm{~b}$ are given.

Our main concern here is to point out that if $Q$ is non-singular, then the stage cost (10) satisfies assumptions A3 and A4a (but not A4b). Using this stage cost in computing $\kappa_{N}(\cdot)$ thus assures that $\mathbf{T}$ is robustly asymptotically stable (but not necessarily finite-time stable) for the closed-loop system.

Furthermore, the stage cost (10) satisfies assumption A5 if $\mathbf{T}$ is a polytope (for proof, see the Appendix). Its use thus allows the robustly stable MPC problem to be solved as a finite-dimensional problem, as will be shown in more detail in the next section.

Remark 5. It is interesting to observe that, provided $Q$ is non-singular, A3 and A4a are satisfied by the new stage cost (10) even if $R$ is singular. The use of the second term is not necessary in guaranteeing robust asymptotic stability; the second term only affects the performance of the closed-loop system.

The second term in the stage cost (10) follows the idea of pre-stabilising predictions in MPC, which was introduced in [26] and developed further by those authors for use in robust MPC [25]; if $Q:=0$ and $R:=I$, then the stage cost (10) is similar to the one used in [25]. However, it is important to note that $A 4 a$ and $A 4 b$ are not satisfied if $Q$ is singular. It is not yet clear how the assumptions in this paper need to be modified in order to use the method proposed in [25] for proving robust stability (in addition to robust attractiveness) of $\mathbf{T}$ when $Q$ is singular and $R$ is non-singular.

Remark 6. Note that the stage cost (9) satisfies A1-A4 but that it does not satisfy A5. However, L(·) as defined in (9) is quasi-convex if $\mathbf{X}, \mathbf{U}$ and $\mathbf{T}$ are convex. In order to compute $\kappa_{N}(x)$ using the approach in [36], one can show that $A 5$ can be relaxed to the condition that $\sum_{k=0}^{N-1} L\left(x_{k}, u_{k}\right)+F\left(x_{N}\right)$ be quasiconvex. Since the sum of quasi-convex functions cannot be guaranteed to be quasi-convex, one cannot

This is a preprint of an article published in International Journal of Robust and Nonlinear Control Copyright (C) 2004 John Wiley \& Sons, Ltd. Int. J. Robust Nonlinear Control 2004; 14:395-413

Prepared using rncauth.cls 
guarantee that $\sum_{k=0}^{N-1} L\left(x_{k}, u_{k}\right)+F\left(x_{N}\right)$ is quasi-convex if $L(\cdot)$ and $F(\cdot)$ are quasi-convex. Therefore, it is not yet known whether A5 can be relaxed in order to allow the stage cost (9) to be used with the approach proposed in [36].

\subsection{Choice of target set}

For methods of computing a terminal constraint $\mathbf{T}$ that satisfies A1, see [20, 22, 23, 34, 36]. However, some further observations regarding $K$ and $\mathbf{T}$ are in order.

The choice of $K$ in (10) is arbitrary, but typically it is chosen such that $A+B K$ has all its eigenvalues inside the unit disk and the control law is optimal via some performance measure. A factor that needs to be taken into consideration is how the choice of $K$ affects the size of $\mathbf{T}$ that one can use. This problem is not yet fully understood, but some proposals have been put forward for computing a sequence of linear control laws and an associated sequence of disturbance invariant sets of increasing size [15].

The exact choice of disturbance invariant $\mathbf{T}$ is perhaps also arbitrary. However, as discussed in detail in $[27,34,36]$, a sensible choice for $\mathbf{T}$ is the minimal disturbance invariant set $\mathcal{F}_{\infty}[22,23]$ for the system $x_{k+1}=(A+B K) x_{k}+w_{k}$, i.e.

$$
\mathcal{F}_{\infty}:=\bigoplus_{i=0}^{\infty}(A+B K)^{i} \mathbf{W},
$$

where $\bigoplus_{i=p}^{q} S_{i}:=\left\{\sum_{i=p}^{q} s_{i} \mid s_{i} \in S_{i}, i=p, \ldots, q\right\}$ denotes the Minkowski (vector) sum [20] of the sets $\left\{S_{p}, \ldots, S_{q}\right\}$. The problem, however, with setting $\mathbf{T}:=\mathcal{F}_{\infty}$ is that the region of attraction $X_{N}$ can be quite small.

One way of enlarging $X_{N}$ is to set $\mathbf{T}$ equal to the maximal disturbance invariant set $\mathcal{O}_{\infty}$ [22, 23] for the system $x_{k+1}=(A+B K) x_{k}+w_{k}$ that is contained inside the constraint-admissible set:

$$
X_{K}:=\{x \in \mathbf{X} \mid K x \in \mathbf{U}\} .
$$

In this case,

$$
\mathcal{O}_{\infty}:=\left\{x_{0} \in X_{K} \mid x_{k+1}=(A+B K) x_{k}+w_{k} \in X_{K}, \forall w_{k} \in \mathbf{W}, k \in \mathbb{N}\right\} .
$$

This choice of target set has the benefit that if the state enters $\mathbf{T}$ in finite time, then one can guarantee that the state of the system $x_{k+1}=A x_{k}+B \Gamma\left(x_{k}\right)+w_{k}$ will robustly converge to the minimal disturbance invariant set $\mathcal{F}_{\infty}$ (this is a consequence of the properties of state trajectories of $x_{k+1}=(A+B K) x_{k}+w_{k}$ that start inside $\left.\mathcal{O}_{\infty}[22, \S 3]\right)$. Recall, however, that with the stage cost (10) one cannot guarantee that the state of the system will enter $\mathbf{T}$ in finite time.

A compromise that results in a smaller $X_{N}$, but still guarantees convergence to the minimal disturbance invariant set $\mathcal{F}_{\infty}$, is to set $\mathbf{T}$ equal to any subset in the interior of $\mathcal{O}_{\infty}$ that is also a disturbance invariant set for the system $x_{k+1}=(A+B K) x_{k}+w_{k}$. Since $\mathbf{T}$ is robustly asymptotically stable, this guarantees that the state of the system $x_{k+1}=A x_{k}+B \kappa_{N}\left(x_{k}\right)+w_{k}$ will enter $\mathcal{O}_{\infty}$ in finite time. As soon as the state enters $\mathcal{O}_{\infty}$, one can switch to the control law $u=K x$, thereby guaranteeing robust convergence of the state of the system $x_{k+1}=(A+B K) x_{k}+w_{k}$ to $\mathcal{F}_{\infty}$. More precisely, if the "dual-mode" control law

$$
\psi(x):= \begin{cases}\kappa_{N}(x) & \text { if } x \in X_{N} \backslash \mathcal{O}_{\infty} \\ K x & \text { if } x \in \mathcal{O}_{\infty}\end{cases}
$$

then the following result follows:

This is a preprint of an article published in International Journal of Robust and Nonlinear Control Copyright (C) 2004 John Wiley \& Sons, Ltd. 
Theorem 2. If $A 1, A 2, A 3$ and $A 4 a$ hold, the eigenvalues of $A+B K$ are strictly inside the unit disk and $\mathbf{T} \subseteq \operatorname{int} \mathcal{O}_{\infty}$, then the minimal disturbance invariant set $\mathcal{F}_{\infty}$ is robustly asymptotically stable for the closed-loop system $x_{k+1}=A x_{k}+B \psi\left(x_{k}\right)+w_{k}$ with a region of attraction $X_{N}$. If, in addition, $\mathcal{F}_{\infty} \subseteq$ int $\mathbf{T}$, then $\mathbf{T}$ is robustly finite-time stable for the closed-loop system $x_{k+1}=A x_{k}+B \psi\left(x_{k}\right)+w_{k}$ with a region of attraction $X_{N}$.

Proof: Robust attraction is a consequence of the above discussion and the fact that $(A+B K)^{k} x \rightarrow 0$ as $k \rightarrow \infty$. Hence, for large $k$, the state trajectories of the system are determined almost entirely by the disturbance sequence and $\mathcal{F}_{\infty}$ is a limit set for the trajectories of $x_{k+1}=(A+B K) x_{k}+w_{k}[22$, $\S 3]$. See also [23] for further details regarding the properties of the maximal and minimal disturbance invariant sets for linear discrete-time systems. Robust stability of $\mathcal{F}_{\infty}$ and $\mathbf{T}$ follows immediately from the fact that $\mu \mathcal{F}_{\infty}$ and $\mu \mathbf{T}$ are disturbance invariant for all $\mu \geq 1$ [10, Prop. 2.1].

Remark 7. Note that Theorem 2 does not require that $A 4 b$ hold.

The new stage cost (10) can be compared to the conventional stage cost $L(x, u):=\|Q x\|_{p}+\|R u\|_{p}$, which is typically used in conventional MPC schemes without disturbances. In the new stage cost (10), deviations of the state trajectory from $\mathbf{T}$ as well as deviations from some "ideal" control law $u=K x$ are penalised, instead of penalising deviations of the state and input from the origin. The minimal disturbance invariant set $\mathcal{F}_{\infty}$ can be thought of as the "origin" of the system. If $\mathbf{T}:=\mathcal{F}_{\infty}$, then one can interpret (10) as penalising deviations from the "origin". Similarly, if $\mathbf{T} \supset \mathcal{F}_{\infty}$, then one can think of the terminal constraint as containing the "origin" (though the stage cost does not penalise deviations from the "origin" anymore).

It is interesting to observe that if $x$ is far from the target set so that $\min _{y \in \mathbf{T}}\|Q(x-y)\|_{p} \approx$ $\|Q x\|_{p}$ and $\|R(u-K x)\|_{p} \approx\|R K x\|_{p}$ for all $u \in \mathbf{U}$, then the new stage cost (10) is such that $L(x, u) \approx\|Q x\|_{p}+\|R K x\|_{p}$ for all $u \in \mathbf{U}$. This is in contrast to having used the conventional cost $L(x, u):=\|Q x\|_{p}+\|R u\|_{p}$ where, for large $x, L(x, u) \approx\|Q x\|_{p}$ for all $u \in \mathbf{U}$. For large $x$, one can interpret the new stage cost as if an extra penalty on the state has been added to the conventional stage cost and it is therefore possible that this additional cost could swamp the original cost on the state, independently of the choice of the input. However, for large states it is quite often the case that the input constraints are active at the optimum, regardless of whether the conventional or new stage cost is used - the use of the new stage cost therefore often does not change the optimal input if the state is far away from the target set. Furthermore, in practice $x$ will not get to be very large because $\mathbf{X}$ is often chosen to constrain the system relatively close to the origin.

It is therefore perhaps more important to consider what happens when the state is close to (but outside) the target set and a subset of the input constraints are inactive at the optimum. In this case, in addition to giving an a priori robust stability and convergence guarantee, the new stage cost (10) has another potential advantage compared to adopting the conventional stage cost. When using a 1-norm or $\infty$-norm stage cost, the problem is often that the optimal receding horizon controller is dead-beat or idle $[3,35]$. This can usually be avoided by using the new stage cost (10), designing an appropriate $K$ and choosing suitable non-singular $Q$ and $R$. The result is that the response of the constrained system $x_{k+1}=A x_{k}+B \Gamma\left(x_{k}\right)+w_{k}$ can be made to be qualitatively close to the response of the unconstrained system $x_{k+1}=(A+B K) x_{k}+w_{k}$ if the initial state is close to the target set $\mathbf{T}$, with only a gradual change in the behaviour of the constrained system as the size of the initial state increases.

This is a preprint of an article published in International Journal of Robust and Nonlinear Control Copyright (c) 2004 John Wiley \& Sons, Ltd.

Prepared using rncauth.cls 


\section{SOLUTION VIA LINEAR PROGRAMMING}

Following the same approach as in [36], let $\mathbf{w}^{\ell}:=\left\{w_{0}^{\ell}, \ldots, w_{N-1}^{\ell}\right\}$ denote an admissible disturbance sequence over the finite horizon $k=0, \ldots, N-1$ and let $\ell \in \mathcal{L}$ index these realisations (this is a slight abuse of notation, because the set of possible realisations is uncountable). Also let $\mathbf{u}^{\ell}:=$ $\left\{u_{0}^{\ell}, \ldots, u_{N-1}^{\ell}\right\}$ denote a control sequence associated with the $\ell^{\prime}$ th disturbance realisation and let $\mathbf{x}^{\ell}:=\left\{x_{0}^{\ell}, \ldots, x_{N}^{\ell}\right\}$ represent the sequence of solutions of the model equation

$$
x_{k+1}^{\ell}=A x_{k}^{\ell}+B u_{k}^{\ell}+w_{k}^{\ell}, \quad \ell \in \mathcal{L}
$$

with $x_{0}^{\ell}=x$, where $x$ denotes the current state.

\subsection{Converting $P_{N}$ to a finite-dimensional problem}

As a first step towards an implementable solution we follow [36] in replacing problem $P_{N}$ by the following equivalent problem, in which the optimisation over feedback policies is achieved by optimising over control sequences, but with the causality constraint (17e) enforced:

Problem 1 (Infinite Dimensional Feedback Min-Max) Given the current state $x$, if

$$
\mathbf{u}_{\infty}:=\left\{u_{k}^{\ell} \mid k=0, \ldots, N-1, \ell \in \mathcal{L}\right\}
$$

find a solution to the problem

$$
\mathbf{u}_{\infty}^{*}(x):=\left(\arg \min _{\mathbf{u}_{\infty}} \max _{\ell \in \mathcal{L}}\left[F\left(x_{N}^{\ell}\right)+\sum_{k=0}^{N-1} L\left(x_{k}^{\ell}, u_{k}^{\ell}\right)\right],\right.
$$

where the optimisation is subject to (16), $x_{0}^{\ell}=x$ for all $\ell \in \mathcal{L}$ and

$$
\begin{array}{rll}
x_{k}^{\ell} \in \mathbf{X}, & k=1, \ldots, N-1, & \forall \ell \in \mathcal{L} \\
u_{k}^{\ell} \in \mathbf{U}, & k=0, \ldots, N-1, & \forall \ell \in \mathcal{L} \\
x_{N}^{\ell} \in \mathbf{T}, & & \forall \ell \in \mathcal{L} \\
x_{k}^{\ell_{1}}=x_{k}^{\ell_{2}} \Rightarrow u_{k}^{\ell_{1}}=u_{k}^{\ell_{2}} & k=0, \ldots, N-1 & \forall \ell_{1}, \ell_{2} \in \mathcal{L} .
\end{array}
$$

As explained in more detail in $[30,33,36]$, this problem is equivalent to the feedback min-max problem $P_{N}$ due to two facts: (i) a different control input sequence is associated with each disturbance sequence, thereby overcoming the problem of open-loop MPC that associates a single control input sequence with all disturbance sequences; (ii) the causality constraint (17e) associates with each $x_{k}^{\ell}$ a single control input, thereby reducing the degrees of freedom and making the control law independent of the control and disturbance sequence taken to reach that state.

Let the finite subset $\mathcal{L}_{v}:=\{1,2, \ldots, V\} \subset \mathcal{L}$, where $V$ is the cardinality of $\mathcal{L}_{V}$, index those disturbance sequences $\mathbf{w}^{\ell}$ that take on values at the vertices of the polytope $\mathbf{W}^{N}$ and consider the following finite dimensional optimisation problem:

Problem 2 (Finite Dimensional Feedback Min-Max) Given the current state $x$, if

$$
\mathbf{u}:=\left\{\mathbf{u}^{1}, \mathbf{u}^{2}, \ldots, \mathbf{u}^{V}\right\}
$$

This is a preprint of an article published in International Journal of Robust and Nonlinear Control Copyright (C) 2004 John Wiley \& Sons, Ltd. Int. J. Robust Nonlinear Control 2004; 14:395-413

Prepared using rncauth.cls 
and

$$
\mathbf{u}^{\ell}:=\left\{u_{0}^{\ell}, u_{1}^{\ell}, \ldots, u_{N-1}^{\ell}\right\}, \quad \forall \ell \in \mathcal{L}_{v},
$$

then find a solution to the problem

$$
\mathbf{u}^{*}(x):=\left(\arg \min _{\mathbf{u}} \max _{\ell \in \mathcal{L}_{v}}\left[F\left(x_{N}^{\ell}\right)+\sum_{k=0}^{N-1} L\left(x_{k}^{\ell}, u_{k}^{\ell}\right)\right],\right.
$$

where the optimisation is subject to $(16), x_{0}^{\ell}=x$ for all $\ell \in \mathcal{L}_{v}$ and

$$
\begin{array}{rll}
x_{k}^{\ell} \in \mathbf{X}, & k=1, \ldots, N-1, & \forall \ell \in \mathcal{L}_{v} \\
u_{k}^{\ell} \in \mathbf{U}, & k=0, \ldots, N-1, & \forall \ell \in \mathcal{L}_{v} \\
x_{N}^{\ell} \in \mathbf{T}, & & \forall \ell \in \mathcal{L}_{v} \\
x_{k}^{\ell_{1}}=x_{k}^{\ell_{2}} \Rightarrow u_{k}^{\ell_{1}}=u_{k}^{\ell_{2}} & k=0, \ldots, N-1, & \forall \ell_{1}, \ell_{2} \in \mathcal{L}_{v} .
\end{array}
$$

At first glance, it might not be obvious how the the causality constraint (18e) can be implemented as a finite set of linear constraints. However, note that for all $k \in\{0, \ldots, N-2\}$ and $\ell_{1}, \ell_{2} \in \mathcal{L}_{v}$, if $x_{0}^{\ell_{1}}=x_{0}^{\ell_{2}}, w_{j}^{\ell_{1}}=w_{j}^{\ell_{2}}$ and $u_{j}^{\ell_{1}}=u_{j}^{\ell_{2}}$ for all $j \in\{0, \ldots, k\}$, then $x_{j}^{\ell_{1}}=x_{j}^{\ell_{2}}$ for all $j \in\{1, \ldots, k+1\}$. Hence one needs to set $u_{k+1}^{\ell_{1}}=u_{k+1}^{\ell_{2}}$ in order to satisfy the causality constraint. Therefore, as discussed in [30,36], the causality constraint (18e) can be replaced by associating the same control input with each node of the resulting extreme disturbance/state trajectory tree. This observation reduces the original number of control inputs that need to be computed from $N v^{N}$ to $1+v+\ldots+v^{N-1}=\left(v^{N}-1\right) /(v-1)$, where $v$ is the number of vertices of $\mathbf{W}$. A similar observation holds for the number of constraints and slack variables that will be introduced below when translating (18) into a finite-dimensional LP.

As a small example, consider the case when $v=2$ and $N=2$. There are $V=v^{N}=4$ extreme disturbance sequences and if $\mathcal{L}_{v}$ has been defined such that $w_{0}^{1}=w_{0}^{2}$ and $w_{0}^{3}=w_{0}^{4}$, then (18e) can be substituted with $u_{0}^{1}=u_{0}^{2}=u_{0}^{3}=u_{0}^{4}, u_{1}^{1}=u_{1}^{2}$ and $u_{1}^{3}=u_{1}^{4}$.

Clearly, the number of decision variables and constraints grows exponentially with the length of the control horizon. Implementing robust MPC formulated along these lines with large control horizons is therefore questionable. However, for some problems the computational complexity might still be acceptable.

If one now lets

$$
\mathbf{u}^{*}(x)=:\left\{\mathbf{u}^{1 *}(x), \mathbf{u}^{2 *}(x), \ldots, \mathbf{u}^{V *}(x)\right\}
$$

and

$$
\mathbf{u}^{\ell *}(x)=:\left\{u_{0}^{\ell *}(x), u_{1}^{\ell *}(x), \ldots, u_{N-1}^{\ell *}(x)\right\}, \quad \forall \ell \in \mathcal{L}_{v},
$$

then one can establish conditions under which the first component of $\mathbf{u}^{1 *}(x)$, denoted by $u_{0}^{1 *}(x)$, is equal to the first element of $\mathbf{u}_{\infty}^{*}(x)$ and hence also equal to $\kappa_{N}(x)$ (note that (18e) ensure that all the $u_{0}^{\ell *}(x), \ell \in \mathcal{L}_{v}$, are equal). As noted in [33, §4.6.3], if the system is linear, $\mathbf{X}, \mathbf{U}, \mathbf{W}$ and $\mathbf{T}$ are polytopes and $F(\cdot)$ and $L(\cdot)$ are convex functions, then using similar convexity arguments as in [36, Thm. 2], it can be shown that the first element of $\mathbf{u}^{1 *}(x)$ is equal to $\kappa_{N}(x)$.

The next result now follows:

This is a preprint of an article published in International Journal of Robust and Nonlinear Control Copyright (c) 2004 John Wiley \& Sons, Ltd. Int. J. Robust Nonlinear Control 2004; 14:395-413

Prepared using rncauth.cls 
Theorem 3 (Robustly Stable Feedback Min-Max MPC/RHC) Suppose Al and A2 are satisfied. If the stage cost is given by (10) and $Q$ is non-singular, then $\kappa_{N}(x)=u_{0}^{1 *}(x)$ and $\mathbf{T}$ is robustly asymptotically stable for the closed-loop system $x_{k+1}=A x_{k}+B \kappa_{N}\left(x_{k}\right)+w_{k}$ with a region of attraction $X_{N}$.

\subsection{Setting up as an LP problem}

In [36] it was proposed that the solution to (18) be computed on-line using standard convex, nonlinear programming solvers. We will now describe how this problem can be solved using linear programming if stage cost (10) is used. This will involve setting up an LP that is equivalent to (18).

Recalling that $F(x):=0$, let the total cost $J\left(x, \mathbf{u}^{\ell}, \mathbf{w}^{\ell}\right)$ for the current state $x$ and a sequence of control inputs $\mathbf{u}^{\ell}$ associated with a given disturbance realisation $\mathbf{w}^{\ell}$ be defined as:

$$
J\left(x, \mathbf{u}^{\ell}, \mathbf{w}^{\ell}\right):=\sum_{k=0}^{N-1} L\left(x_{k}^{\ell}, u_{k}^{\ell}\right) .
$$

As in [36], the optimisation (18) can be written as

$$
\min _{\mathbf{u} \in \mathbf{C}(x)} \max _{\ell \in \mathcal{L}_{v}} J\left(x, \mathbf{u}^{\ell}, \mathbf{w}^{\ell}\right),
$$

where $\mathbf{C}(x)$ is a polytope implicitly defined by the constraints in (18). Clearly, (19) is equivalent to the following convex program:

$$
\min _{\mathbf{u}, \gamma}\left\{\gamma \mid \mathbf{u} \in \mathbf{C}(x), J\left(x, \mathbf{u}^{\ell}, \mathbf{w}^{\ell}\right) \leq \gamma, \forall \ell \in \mathcal{L}_{v}\right\} .
$$

Before proceeding, note that if one uses the stage cost (10) with $p=1$ then the value of $\min _{u \in \mathbf{U}} L(x, u)$ can be computed by solving the LP

$$
\min _{u \in \mathbf{U}} L(x, u)=\min _{u, y, \alpha, \beta, \gamma} \gamma
$$

subject to

$$
-\alpha \leq Q(x-y) \leq \alpha, \quad-\beta \leq R(u-K x) \leq \beta, \quad u \in \mathbf{U}, \quad y \in \mathbf{T}, \quad \mathbf{1}^{\prime} \alpha+\mathbf{1}^{\prime} \beta \leq \gamma,
$$

where $\alpha \in \mathbb{R}^{n}, \beta \in \mathbb{R}^{m}$ and the vector $\mathbf{1}:=[1,1, \ldots, 1]^{\prime}$ has appropriate length.

The above procedure is fairly standard and has been used in converting standard and open-loop minmax MPC problems with 1-norm and $\infty$-norm costs to linear programs [1, 2, 3, 4, 14, 30, 35, 40]. We now use it to set up an LP that is equivalent to (20). Let

$$
J\left(x, \mathbf{u}^{\ell}, \mathbf{w}^{\ell}\right):=\min _{\mathbf{y}^{\ell}} \sum_{k=0}^{N-1}\left\|Q\left(x_{k}^{\ell}-y_{k}^{\ell}\right)\right\|_{1}+\left\|R\left(u_{k}^{\ell}-K x_{k}^{\ell}\right)\right\|_{1},
$$

and $\mathbf{y}^{\ell}, \mu^{\ell}, \eta^{\ell}$ and $\mathbf{y}, \mu, \eta$ be defined similarly to $\mathbf{u}^{\ell}$ and $\mathbf{u}$. It now follows that (20) (and hence (18)) is equivalent to

$$
\min _{\mathbf{u}, \mathbf{y}, \mu, \eta, \gamma} \gamma
$$

This is a preprint of an article published in International Journal of Robust and Nonlinear Control Copyright (C) 2004 John Wiley \& Sons, Ltd. Int. J. Robust Nonlinear Control 2004; 14:395-413

Prepared using rncauth.cls 
subject to

$$
\begin{array}{llrl}
x_{k+1}^{\ell}=A x_{k}^{\ell}+B u_{k}^{\ell}+w_{k}^{\ell}, & x_{0}^{\ell}=x, \quad k=0, \ldots, N-1, & \forall \ell \in \mathcal{L}_{v} \\
& \begin{array}{c}
x_{k}^{\ell} \in \mathbf{X}, \quad k=1, \ldots, N-1, \\
x_{N}^{\ell} \in \mathbf{T},
\end{array} & \forall \ell \in \mathcal{L}_{v} \\
& \quad \quad k=0, \ldots, N-1, & \forall \ell \in \mathcal{L}_{v} \\
x_{k}^{\ell_{1}}=x_{k}^{\ell_{2}} \Rightarrow u_{k}^{\ell_{1}}=u_{k}^{\ell_{2}} & \ell_{2} \in \mathcal{L}_{v} \\
-\mu_{k}^{\ell} \leq Q\left(x_{k}^{\ell}-y_{k}^{\ell}\right) \leq \mu_{k}^{\ell}, & y_{k}^{\ell} \in \mathbf{T}, \quad k=0, \ldots, N-1, & \forall \ell \in \mathcal{L}_{v} \\
-\eta_{k}^{\ell} \leq R\left(u_{k}^{\ell}-K x_{k}^{\ell}\right) \leq \eta_{k}^{\ell}, & u_{k}^{\ell} \in \mathbf{U}, & k=0, \ldots, N-1, & \forall \ell \in \mathcal{L}_{v} \\
\sum_{k=0}^{N-1} \mathbf{1}^{\prime} \mu_{k}^{\ell}+\mathbf{1}^{\prime} \eta_{k}^{\ell} \leq \gamma, & & \forall \ell \in \mathcal{L}_{v} .
\end{array}
$$

Remark 8. Note that a minimiser to the LP (21) exists if $X_{N}$ is non-empty and the current state $x \in X_{N}$. This is because the sets $\mathbf{U}$ and $\mathbf{T}$ are compact and the variables $\mu, \eta$ and $\gamma$ are bounded below by zero. This justifies the assumption made in Section 2 regarding the existence of a minimiser to problem $P_{N}(x)$.

Remark 9. Note that it is also possible to convert the feedback min-max MPC problem to an LP if $p=\infty$ is chosen in the stage cost (10). This is achieved in a similar fashion as above by noting that if $L(x, u):=\min _{y \in \mathbf{T}}\|Q(x-y)\|_{\infty}+\|R(u-K x)\|_{\infty}$, then

$$
\min _{u \in \mathbf{U}} L(x, u)=\min _{u, y, \alpha, \beta, \gamma} \gamma
$$

subject to

$$
-1 \alpha \leq Q(x-y) \leq \mathbf{1} \alpha, \quad-\mathbf{1} \beta \leq R(u-K x) \leq \mathbf{1} \beta, \quad u \in \mathbf{U}, \quad y \in \mathbf{T}, \quad \alpha+\beta \leq \gamma,
$$

where $\alpha \in \mathbb{R}, \beta \in \mathbb{R}$ and the vector $\mathbf{1}:=[1,1, \ldots, 1]^{\prime}$ has appropriate length.

It is interesting to observe that the use of the $\infty$-norm results in less variables and constraints than in the case of the 1-norm. The former choice of norm is therefore probably preferred if computational speed is an issue. However, the latter norm might be preferred if a control action is sought that is closer to having used the quadratic norm, as in conventional MPC.

\subsection{Explicit solution of the RHC law via parametric programming}

The development in the previous section allows the on-line solution of the robust MPC problem, providing that the available computing resources and the required update interval are such that the LP can be solved quickly enough. If this is not possible, an alternative is to pre-compute the solution, to store this solution in a database, and to read out the appropriate part of the solution (which can be done relatively quickly) as required.

By substituting (21b) into the rest of the constraints it is possible to show, as in [3, 6], that (21) can be written in the form

$$
\min _{\theta}\left\{c^{\prime} \theta \mid F \theta \leq g+G x\right\}
$$

where $\theta$ is the decision variable and consists of the non-redundant components of $(\mathbf{u}, \mathbf{y}, \mu, \eta, \gamma)$; the vectors $c, g$ and matrices $F, G$ are of appropriate dimensions and do not depend on $x$. The key observation here is that the constraints are dependent on the current state $x$ in the affine manner shown.

This is a preprint of an article published in International Journal of Robust and Nonlinear Control Copyright (C) 2004 John Wiley \& Sons, Ltd. 
This means that the feedback min-max problem falls into the class of multi-parametric linear programs (mp-LPs) $[8,18]$, where each component of $x$ represents a parameter that will affect the solution. This class of problems can be solved off-line for all allowable values of $x$ and results in a piecewise affine expression for the solution in terms of $x[8,18]$.

The polyhedron $X_{N}=\left\{x \in \mathbb{R}^{n} \mid \exists \theta: F \theta \leq g+G x\right\}$ is clearly the set of states for which a solution to (22) (and problem $P_{N}$ ) exists. Given a polytope of states $\mathcal{X} \subseteq X_{N}$ and using the algorithm described in [8], one can compute the explicit solution of the feedback min-max control law for all $x \in \mathcal{X}$. The resulting feedback min-max RHC law is then of the following piecewise affine form:

$$
\kappa_{N}(x)=K_{i} x+h_{i}, \forall x \in \mathcal{R}_{i},
$$

where each $K_{i} \in \mathbb{R}^{m \times n}$ and $h_{i} \in \mathbb{R}^{m}$ are associated with a polytope $\mathcal{R}_{i}$; each $\mathcal{R}_{i}$ is equal to the intersection of $\mathcal{X}$ and the closure of a so-called critical region (a critical region is the set of all parameters $x$ such that a given set of constraints are active at the optimum of (22); see [8] for a rigorous definition). The set of polytopes $\left\{\mathcal{R}_{i}\right\}$ have mutually disjoint interiors and $\mathcal{X}=\bigcup_{i} \mathcal{R}_{i}$. All that is required on-line is to determine which polytope $\mathcal{R}_{i}$ contains the current state (see $[7,38]$ for efficient methods) and then compute the control action using only matrix multiplication and addition.

Remark 10. The solution to the control law presented in this paper is of the same piecewise affine structure as the one given in [4, 5]. However, the derivation in [4] is based on dynamic programming and requires the solution of $2 N$ multi-parametric mixed-integer linear programs (mp-MILPs). By exploiting the convex, piecewise affine nature of the optimal cost, this has since been improved to solving $N$ mp-LPs [5]. In contrast, the results presented in this paper requires the solution of a single mp-LP, though this is perhaps of more significance for the on-line computation of the MPC solution than for off-line pre-computation of the RHC law.

\section{EXAMPLES}

The following two examples were implemented in Matlab 6.5. The mp-LP solver was implemented using the algorithm described in [8] and the LP solver provided with $c d d / c d d+[17]$. All computations were performed on an AMD Athlon 1.9GHz processor.

\subsection{Case $n=1$}

The first example is taken from $[4,36]$. The system is given by

$$
x_{k+1}=x_{k}+u_{k}+w_{k},
$$

with $\mathbf{U}:=\mathbb{R}$ and

$$
\mathbf{X}:=\{x \in \mathbb{R} \mid-1.2 \leq x \leq 2\}, \mathbf{T}:=\{x \in \mathbb{R}|| x \mid \leq 1\}, \mathbf{W}:=\{w \in \mathbb{R}|| w \mid \leq 1\} .
$$

For an initial comparison, the same stage and terminal costs as in [4] were used, i.e.

$$
L(x, u):=|Q x|+|R u|, F(x):=0, \forall x \in \mathbb{R}^{n}, u \in \mathbb{R}^{m}
$$

with $Q=1$ and $R=10$. With $N=2$ and $\mathcal{X}:=\{x \in \mathbb{R} \mid-1.2 \leq x \leq 2\}$, by solving a single mp-LP as described in this paper, the robust RHC law $\kappa_{N}(\cdot)$ was found to be

$$
\kappa_{N}(x)=-x \text { if }-1.2 \leq x \leq 2,
$$

This is a preprint of an article published in International Journal of Robust and Nonlinear Control Copyright (c) 2004 John Wiley \& Sons, Ltd. Int. J. Robust Nonlinear Control 2004; 14:395-413

Prepared using rncauth.cls 
which is the same as [4, Eqn. 24].

The computation of $\kappa_{N}(\cdot)$ took $55 \mathrm{~ms}$. This is a considerable improvement to the $55 \mathrm{~s}$ it took in [4] to solve $4 \mathrm{mp}$-MILPs on a similarly-specified computer and is comparable with the $1.3 \mathrm{~s}$ it took in [5] to solve the same problem using 2 mp-LPs.

When the new stage cost (10) was used, i.e.

$$
L(x, u):=\min _{y \in \mathbf{T}}|Q(x-y)|+|R(u-K x)|
$$

with $K:=-1$ (as proposed in $[36, \S \mathrm{F}])$, the robust control law $\kappa_{N}(\cdot)$ was computed in $68 \mathrm{~ms}$ and found to be the same as in (23).

\subsection{Case $n=2$}

For the second example, the system is given by

with

$$
x_{k+1}=\left[\begin{array}{ll}
1 & 0.8 \\
0 & 0.7
\end{array}\right] x_{k}+\left[\begin{array}{l}
0 \\
1
\end{array}\right] u_{k}+w_{k}
$$

$$
\mathbf{X}:=\left\{x \in \mathbb{R}^{2} \mid\|x\|_{\infty} \leq 10\right\}, \mathbf{W}:=\left\{w \in \mathbb{R}^{2} \mid\|w\|_{\infty} \leq 0.1\right\}, \mathbf{U}:=\{u \in \mathbb{R} \mid-3 \leq u \leq 3\} .
$$

Given $K:=-[11]$, the target set was chosen to be the maximal disturbance invariant set $\mathcal{O}_{\infty}$ contained inside $X_{K}:=\{x \in \mathbf{X} \mid K x \in \mathbf{U}\}$ for the closed-loop system $x_{k+1}=(A+B K) x_{k}+w_{k}$, i.e.

$$
\mathbf{T}:=\mathcal{O}_{\infty}=\left\{x \in \mathbb{R}^{2} \mid-\left[\begin{array}{c}
3 \\
2.8 \\
2.75
\end{array}\right] \leq\left[\begin{array}{cc}
1 & 1 \\
0 & 0.5 \\
0.5 & 0.15
\end{array}\right] x \leq\left[\begin{array}{c}
3 \\
2.8 \\
2.75
\end{array}\right]\right\} .
$$

The stage cost was chosen to be

$$
L(x, u):=\min _{y \in \mathbf{T}}\|Q(x-y)\|_{\infty}+\|R(u-K x)\|_{\infty},
$$

with $Q=I$ and $R=0.1$. The control horizon was set to $N=2$ and $\mathcal{X}:=X_{N} \cap \mathbf{X}$ was computed using the software developed in [20].

The LP that solves the feedback min-max MPC problem has 220 inequalities (of which only 105 are non-redundant) and 42 decision variables. The computation of the explicit expression for the RHC law $\kappa_{N}(\cdot)$ was completed in under $23 \mathrm{~s}$ ( since by far most of the computational effort actually goes into removing redundant inequalities from the newly computed polytopes that partition the state space, it is expected that this time can be reduced by a few orders of magnitude using a state-of-the-art LP solver). The polytopes that define the explicit solution of the associated mp-LP are shown in Figure 1 (in order to save space, the expressions for the associated polytopes are not listed). Though 71 separate regions were computed, it was found that only 7 distinct affine control laws were defined over different parts of $\mathcal{X}$ (polytopes with the same affine control law are plotted with the same shade in Figure 1). Postprocessing might therefore reduce the number of regions that need to be stored on-line. The 7 affine control laws that, together with the polytopes shown in Figure 1, define $\kappa_{N}(\cdot)$ are:

$$
\begin{aligned}
\kappa_{N}^{1,2}(x) & =\left[\begin{array}{ll}
0 & 0
\end{array}\right] x \pm 3 \\
\kappa_{N}^{3,4}(x) & =\left[\begin{array}{ll}
0 & -0.7
\end{array}\right] x \pm 5.5, \\
\kappa_{N}^{5,6}(x) & =\left[\begin{array}{ll}
-1 & -1.5
\end{array}\right] x \pm 2.8, \\
\kappa_{N}^{7}(x) & =\left[\begin{array}{ll}
-1 & -1
\end{array}\right] x .
\end{aligned}
$$

This is a preprint of an article published in International Journal of Robust and Nonlinear Control Copyright (C) 2004 John Wiley \& Sons, Ltd. Int. J. Robust Nonlinear Control 2004; 14:395-413

Prepared using rncauth.cls 


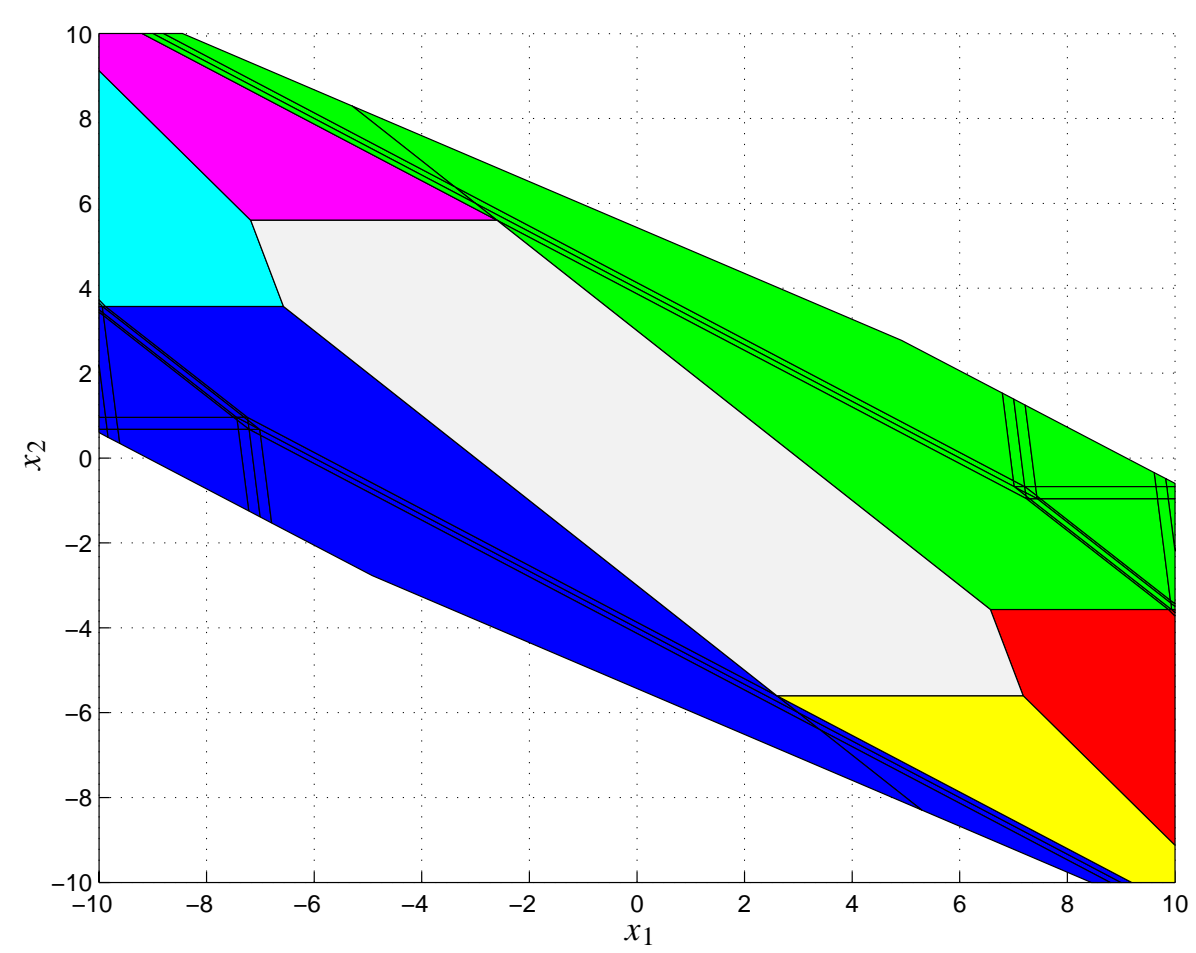

Figure 1. The polytopes that define the explicit expression for $\kappa_{N}(\cdot)$ for the second example

Finally, Figure 2 shows part of the response of the closed-loop system $x_{k+1}=A x_{k}+B \Gamma\left(x_{k}\right)+w_{k}$ to a random, persistent disturbance satisfying $w_{k} \in \mathbf{W}$ for all $k \in \mathbb{N}$, starting from initial state $x_{0}=\left[\begin{array}{ll}10 & -10\end{array}\right]^{\prime}$. As can be seen, the presence of the persistent disturbance prevents the state of the system from converging to the origin. Note that in this example the state enters $\mathbf{T}$ in finite time, despite the fact that only robust asymptotic convergence to the target set $\mathbf{T}$ was guaranteed. Recall also that $\Gamma(\cdot)$ and $\mathbf{T}$ have been defined such that if the state enters $\mathbf{T}$ in finite time, then the state of the system is guaranteed to remain inside $\mathbf{T}$ for all future admissible disturbance sequences. Furthermore, using the arguments presented in Section 3, it follows that if the state enters $\mathbf{T}$ in finite time, then the control law $\Gamma(\cdot)$ is such that the state of the closed-loop system will robustly converge to the minimal disturbance invariant set $\mathcal{F}_{\infty}$. Finally, if the state enters $\mathcal{F}_{\infty}$ in finite time, then the trajectory of the closed-loop system $x_{k+1}=A x_{k}+B \Gamma\left(x_{k}\right)+w_{k}$ is guaranteed to remain inside $\mathcal{F}_{\infty}$.

\section{CONCLUSIONS}

Robust MPC requires optimisation over feedback policies, rather than the more traditional optimisation over open-loop sequences, if excessive conservativeness, and hence infeasibility and/or instability, is to be avoided. But this is difficult to implement with reasonable computational effort, and hence its practicality is questionable, particularly if on-line optimisation in real-time is envisaged.

In this paper we have introduced a new stage cost, that allows one to compute the solution of the full

This is a preprint of an article published in International Journal of Robust and Nonlinear Control Copyright (c) 2004 John Wiley \& Sons, Ltd.

Int. J. Robust Nonlinear Control 2004; 14:395-413

Prepared using rncauth.cls 


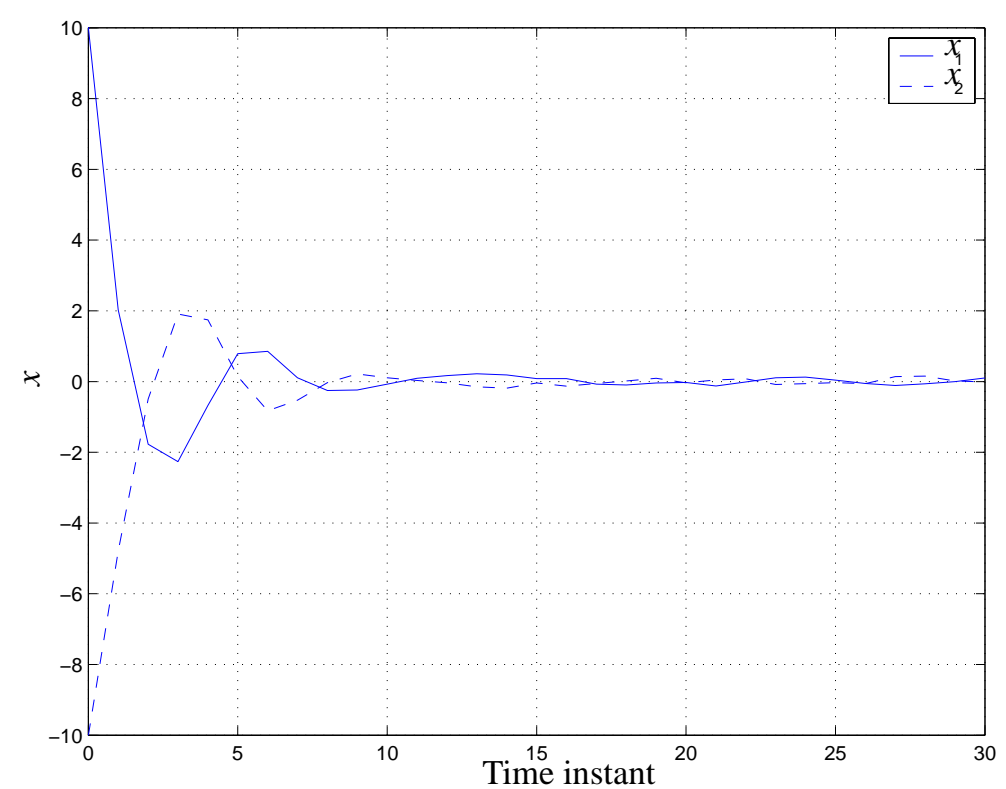

Figure 2. Closed-loop response of the second example to a random, persistent disturbance

robust MPC problem - that is, optimisation over feedback policies with guaranteed robust asymptotic stability of the target set in the face of persistent disturbances - using only one LP. A detailed computational comparison of the competing proposals is not straightforward, however, because the dimensions of the optimisations involved vary in complicated ways. It is therefore not yet possible to say conclusively which scheme will be more efficient for on-line implementation, or which one would be preferred for off-line pre-computation. The answers may well depend on problem-specific details.

Finally, it still remains to be determined, via extensive analysis, simulations and practical applications, exactly when and why it makes sense to use the the new stage cost proposed in this paper, rather than using the conventional type of stage cost or the one proposed in [21].

\section{APPENDIX: PROOF THAT (10) IS CONVEX}

We need to prove that

$$
L\left(\lambda x_{1}+[1-\lambda] x_{2}, \lambda u_{1}+[1-\lambda] u_{2}\right) \leq \lambda L\left(x_{1}, u_{1}\right)+[1-\lambda] L\left(x_{2}, u_{2}\right)
$$

for all $\lambda \in[0,1]$. Note that the proof relies on the convexity of $\mathbf{T}$ and that it is easy to demonstrate that $L(\cdot)$ is not convex if $\mathbf{T}$ is not convex.

\section{Proof:}

$$
\begin{aligned}
& L\left(\lambda x_{1}+[1-\lambda] x_{2}, \lambda u_{1}+[1-\lambda] u_{2}\right)= \\
& \quad \min _{y \in \mathbf{T}}\left\|Q\left(\lambda x_{1}+[1-\lambda] x_{2}-y\right)\right\|_{p}+\left\|R\left(\lambda u_{1}+[1-\lambda] u_{2}-K\left\{\lambda x_{1}+[1-\lambda] x_{2}\right\}\right)\right\|_{p}
\end{aligned}
$$

This is a preprint of an article published in International Journal of Robust and Nonlinear Control Copyright (c) 2004 John Wiley \& Sons, Ltd. 
Let

$$
y_{i}:=\arg \min _{y \in \mathbf{T}}\left\|Q\left(x_{i}-y\right)\right\|_{p}
$$

and consider the first term on the right hand-side of (25), noting that $\lambda y_{1}+[1-\lambda] y_{2} \in \mathbf{T}$ since $\mathbf{T}$ is convex:

$$
\begin{aligned}
\min _{y \in \mathbf{T}}\left\|Q\left(\lambda x_{1}+[1-\lambda] x_{2}-y\right)\right\|_{p} \leq \| Q\left(\lambda x_{1}+\right. & {\left.[1-\lambda] x_{2}-\lambda y_{1}-[1-\lambda] y_{2}\right) \|_{p} } \\
& \leq \lambda\left\|Q\left(x_{1}-y_{1}\right)\right\|_{p}+[1-\lambda]\left\|Q\left(x_{2}-y_{2}\right)\right\|_{p}
\end{aligned}
$$

(Minkowski's inequality). Now consider the second term on the right hand-side of (25):

$$
\begin{array}{r}
\left\|R\left(\lambda u_{1}+[1-\lambda] u_{2}-K\left\{\lambda x_{1}+[1-\lambda] x_{2}\right\}\right)\right\|_{p}=\left\|\lambda R\left(u_{1}-K x_{1}\right)+[1-\lambda] R\left(u_{2}-K x_{2}\right)\right\|_{p} \\
\leq \lambda\left\|R\left(u_{1}-K x_{1}\right)\right\|_{p}+[1-\lambda]\left\|R\left(u_{2}-K x_{2}\right)\right\|_{p}
\end{array}
$$

Adding together (26) and (27) proves (24).

\section{ACKNOWLEDGEMENTS}

The authors would like to thank David Mayne for valuable feedback and discussions during the preparation of this paper, especially with regards Section 3.2. Many thanks also to Colin Jones for helping compute the explicit solutions in Section 5.

\section{REFERENCES}

1. Allwright JC. On min-max model-based predictive control. In Advances in Model-Based Predictive Control, Clarke D (ed) Oxford University Press, 1994; 415-426.

2. Allwright JC, Papavasiliou GC. On linear programming and robust model-predictive control using impulse-responses. Systems \& Control Letters 1992; 18:159-164.

3. Bemporad A, Borrelli F, Morari M. Model predictive control based on linear programming — The explicit solution. IEEE Transactions on Automatic Control 2002; 47(12):1974-1985.

4. Bemporad A, Borrelli F, Morari M. Robust model predictive control: Piecewise linear explicit solution. In Proceedings of the European Control Conference 2001. Porto, Portugal.

5. Bemporad A, Borrelli F, Morari M. Min-max control of constrained uncertain discrete-time linear systems. To appear in IEEE Transactions on Automatic Control.

Technical report available from http://control.ee.ethz.ch [17 September 2003].

6. Bemporad A, Morari M, Dua V, Pistikopoulos EN. The explicit linear quadratic regulator for constrained systems. Automatica 2002; 38(1):3-20.

7. Borrelli F, Baotic M, Bemporad A, Morari M. Efficient on-line computation of constrained optimal control. In Proceedings of the 40th IEEE Conference on Decision and Control 2001. Orlando, Florida, USA.

8. Borrelli F, Bemporad A, Morari M. A geometric algorithm for multi-parametric linear programming. To appear in Journal of Optimization Theory and Applications.

Technical report available from http://control.ee.ethz.ch [17 September 2003].

9. Blanchini F. Minimum-time control for uncertain discrete-time linear systems. In Proceedings of the 31st IEEE Conference on Decision and Control 1992. Tucson AZ, USA; 2629-2634.

10. Blanchini F. Ultimate boundedness control for uncertain discrete-time systems via set-induced Lyapunov functions. IEEE Transactions on Automatic Control 1994; 39(2):428-433.

11. Blanchini F. Set invariance in control. Automatica 1999; 35:1747-1767.

12. Bertsekas DP, Rhodes IB. On the minimax reachability of target sets and target tubes. Automatica 1971; 7:233-247.

13. Bertsekas DP, Rhodes IB. Sufficiently informative functions and the minimax feedback control of uncertain dynamic systems. IEEE Transactions on Automatic Control 1973; AC-18:117-124.

This is a preprint of an article published in International Journal of Robust and Nonlinear Control Copyright (c) 2004 John Wiley \& Sons, Ltd.

Prepared using rncauth.cls

Int. J. Robust Nonlinear Control 2004; 14:395-413 
14. Campo PJ, Morari M. Robust model predictive control. In Proceedings of the American Control Conference 1987. Green Valley AZ, USA; 1021-1026.

15. Cannon M, Deshmukh V, Kouvaritakis B. Nonlinear model predictive control with polytopic invariant sets. In Proceedings of the 15th IFAC World Congress on Automatic Control 2002. Barcelona, Spain.

16. Delfour MC, Mitter SK. Reachability of perturbed systems and min sup problems. SIAM Journal of Control 1969; 47(4):521-533.

17. Fukuda K. cdd and cddplus Homepage. http://www.cs.mcgill.ca/ fukuda/soft/cdd_home/cdd.html [17 September 2003].

18. Gal T. Postoptimal Analyses, Parametric Programming, and Related Topics, (2nd edn). De Gruyter, 1995.

19. Glover JD, Schweppe FC. Control of linear dynamic systems with set constrained disturbances. IEEE Transactions on Automatic Control 1971; AC-16(5):411-423.

20. Kerrigan EC. Robust Constraint Satisfaction: Invariant Sets and Predictive Control. PhD Thesis. University of Cambridge, UK, 2000. Thesis and associated Matlab Invariant Set Toolbox available for download at http://www-control.eng.cam.ac.uk/eck21 [17 September 2003].

21. Kerrigan EC, Mayne DQ. Optimal control of constrained, piecewise affine systems with bounded disturbances. In Proceedings of the 41st IEEE Conference on Decision and Control 2002. Las Vegas NV, USA.

22. Kolmanovsky I, Gilbert EG. Maximal output admissible sets for discrete-time systems with disturbance inputs. In Proceedings of the American Control Conference 1995. Seattle WA, USA; 1995-2000.

23. Kolmanovsky I, Gilbert EG. Theory and computation of disturbance invariant sets for discrete-time linear systems. Mathematical Problems in Engineering: Theory, Methods and Applications 1998; 4:317-367.

24. Kothare MV, Balakrishnan V, Morari M. Robust constrained model predictive control using linear matrix inequalities. Automatica 1996; 32(10):1361-79.

25. Kouvaritakis B, Rossiter JA, Schuurmans J. Efficient robust predictive control. IEEE Transactions on Automatic Control $2000 ;$ 45(8): $1545-1549$.

26. Kouvaritakis B, Rossiter JA. Stable generalized predictive control. In IEE Proceedings, Part D 1992; 139:349-362.

27. Langson W, Chryssochoos I, Mayne D. Robust model predictive control using tubes. Technical Report EEE/C\&P/DQM/11/2001. Imperial College London, UK.

28. Lee JH, Yu Z. Worst-case formulations of model predictive control for systems with bounded parameters. Automatica 1997; 33(5):763-781.

29. Lee YI, Kouvaritakis B. A linear programming approach to constrained robust predictive control. IEEE Transactions on Automatic Control 2000; 45(9):1765-1770.

30. Maciejowski JM. Predictive Control with Constraints. Prentice Hall, UK, 2002.

31. Mayne DQ. Nonlinear model predictive control: An assessment. In Fifth International Conference on Chemical Process Control (CPC V) Kantor JC, Garcia CE, Carnahan B (eds). Tahoe City CA, USA, 1996. CACHE, AIChE.

32. Mayne DQ. Control of constrained dynamic systems. European Journal of Control 2001; 7:87-99.

33. Mayne DQ, Rawlings JB, Rao CV, Scokaert POM. Constrained model predictive control: Stability and optimality. Automatica 2000; 36:789-814.

34. Mayne DQ, Schroeder WR. Robust time-optimal control of constrained linear systems. In Automatica 1997; 33(12):21032118.

35. Rao CV, Rawlings JB. Linear programming and model predictive control. Journal of Process Control 2000; 10:283-289.

36. Scokaert POM, Mayne DQ. Min-max feedback model predictive control. IEEE Transactions on Automatic Control 1998; 43(8):1136-1142.

37. Scokaert POM, Mayne DQ, Rawlings JB. Suboptimal model predictive control (Feasibility implies stability). IEEE Transactions on Automatic Control 1999; 44(3):648-654.

38. Tøndel P, Johansen TA, Bemporad A. Evaluation of piecewise affine control via binary search tree. Automatica 2003. 39:945-950.

39. Witsenhausen HS. A minimax control problem for sampled linear systems. IEEE Transactions on Automatic Control 1968; 13(1):5-21.

40. Zheng ZQ. Robust Control of Systems Subject to Constraints. PhD Thesis. California Institute of Technology, Pasadena CA, USA, 1995.

This is a preprint of an article published in International Journal of Robust and Nonlinear Control Copyright (C) 2004 John Wiley \& Sons, Ltd.

Int. J. Robust Nonlinear Control 2004: 14:395-413

Prepared using rncauth.cls 